Abhandlung

Prof. Dr. Volker Haas

\title{
Erscheinungsformen und Problematik der teilweisen Mittäterschaft
}

Volker Haas: Der Autor ist Inhaber eines Lehrstuhls für Strafrecht und Strafprozessrecht am Institut für Deutsches, Europäisches und Internationales Strafrecht und Strafprozessrecht der juristischen Fakultät der Universität Heidelberg.

\section{Einleitung}

Es ist ein verbreitetes Vorurteil, dass man im Strafrecht keine neuen Probleme mehr entdecken bzw. nichts mehr Neues sagen könne. Wenn man jedoch genau denkt, dann zeigt sich, dass in vielen Bereichen die Dogmatik durchaus noch modifiziert oder präzisiert werden kann. Vielfach sind es konkrete Fälle, anhand derer der Revisions- bzw. Präzisierungsbedarf offenbar wird. Der Einzelfall wird auf diese Weise zum Katalysator des dogmatischen Fortschritts. Im Folgenden soll dargelegt werden, dass im Bereich bzw. Umfeld dessen, was man teilweise Mittäterschaft $\mathrm{zu}$ nennen pflegt, eine bisher noch kaum bzw. überhaupt noch nicht diskutierte Problematik ihrer Beantwortung harrt und dass ihre Beantwortung die herrschende Meinung womöglich dazu zwingt, im Rahmen des Beteiligungssystems den Anwendungsbereich und die Voraussetzungen der Rechtsfiguren der Mittäterschaft und der mittelbaren Täterschaft $\mathrm{zu}$ überdenken und neu $\mathrm{zu}$ justieren. Zunächst sollen kurz die dogmatischen Eckpunkte umrissen werden, die von der Rechtsprechung und der herrschenden Meinung bisher im Hinblick auf die teilweise Mittäterschaft formuliert worden sind. Sodann werden die kontroversen bzw. noch kaum oder nicht beachteten Fälle ins Blickfeld gerückt.

\section{Zu den Voraussetzungen der teilweisen Mittäterschaft}

Wenn im strafrechtlichen Schrifttum von teilweiser Mittäterschaft gesprochen wird, dann ist der Fall gemeint, dass die Beteiligten aufgrund ihres Verhaltens einen Tatbestand mittäterschaftlich verwirklichen, weitere Tatbestände infolge dieses Verhaltens aber nur von einem
Teil der Beteiligten erfüllt werden. ${ }^{1}$ Aufgrund der Tatbestandsbezogenheit der Mittäterschaft wird diese Aufspaltung grundsätzlich für möglich gehalten. ${ }^{2}$ Das häufigst genannte Beispiel betrifft den Mord nach $\S 211$ StGB und den Totschlag nach $\S 212$ StGB. Die Möglichkeit einer Aufspaltung ist vollkommen unproblematisch, wenn derjenige Beteiligte, dessen Verhalten dem Tatgenossen außerordentlich zugerechnet werden soll, einen zusätzlichen Tatbestand verwirklicht, wie zum Beispiel in dem Fall, in dem A aufgrund eines gemeinsamen Tatentschlusses mit B das Opfer tötet und dabei als Einziger ein Mordmerkmal erfüllt, während B einen sonstigen hinreichenden Tatbeitrag leistet. A hat einen Mord gemäß § 211 StGB begangen. Er ist unmittelbarer Täter gemäß § 25 I 1. Var StGB, da er den Tatbestand eigenhändig verwirklicht hat. Folgt man der herrschenden Meinung, die nicht verlangt, dass jeder der Mittäter ein Tatbestandsmerkmal verwirklichen muss, ${ }^{3}$ hat $\mathrm{B}$ in diesem Fall einen Totschlag in Mittäterschaft gemäß den $\S \S 212,25$ II StGB begangen. Die Annahme einer Mittäterschaft wird nicht dadurch infrage gestellt, dass A einen Mord nach $\S 211$ StGB begangen hat, da in diesem Tatbestand der Totschlag nach $\S 212$ StGB enthalten ist. ${ }^{4}$

Ungleich problematischer ist der umgekehrte Fall, den der Bundesgerichtshof zu entscheiden hatte: Der Sohn erschlug die Tante seiner Mutter mit einer Bleikristallvase ohne Habgier und aufgrund einer hirnorganischen Schädigung und einer starken affektiven Erregung ohne das Bewusstsein, den Schlaf des Opfers zur Begehung der Tat auszunutzen. Bei seiner Mutter hingegen, die ihn eindringlich zu dieser Art der Tatausführung aufgefordert und die Tatausführung von der Tür her überwacht hatte, lagen die

1 Roxin, Strafrecht Allgemeiner Teil, Bd. 2, 2003, § 25 Rn. 235; Schünemann, Leipziger Kommentar, Bd. 1, 12. Aufl., 2007, § 25 Rn. 169; Heine, Schönke/Schröder, 28. Aufl., 2010, § 25 Rn. 87/88.

2 Schünemann, LK (Fn. 1), § 25 Rn. 169; Kühl, Strafrecht Allgemeiner Teil, 7. Aufl., 2012, § 20 Rn. 102; Wessels/Beulke, Strafrecht Allgemeiner Teil, 42. Auf., 2012, Rn. 531.

3 Siehe die Übersicht über den Meinungsstand bei Heine, (Fn. 1), Sch/Sch, § 25 Rn. $64 \mathrm{ff}$.

4 Vgl. Beulke/Hillenkamp, JuS 1975, S. 311, 313; Hassemer, JuS 1990, S. $148 \mathrm{f}$. 
Voraussetzungen der Habgier und der Heimtücke vor. ${ }^{5}$ Der Sohn, der lediglich einen Totschlag begangen hatte, war unmittelbarer Täter, weil ausschließlich er die tatbestandliche Ausführungshandlung vollzogen hatte. Nur bei der Mutter, bei der es einer außerordentlichen Zurechnung des Verhaltens ihres Sohnes bedurfte, waren die Voraussetzungen des § 211 StGB erfüllt. Hinter der eher »harmlos« anmutenden Definition der teilweisen Mittäterschaft verbirgt sich damit das Problem, ob die strafbarkeitsbegründende Funktion des $\S 25$ II StGB im Hinblick auf einen bestimmten Straftatbestand auch dann eingreift, wenn die außerordentliche Verhaltenszurechnung der Mittäterschaft dazu führen würde, dass der Zurechnungsadressat diesen Straftatbestand verwirklichen würde, nicht aber derjenige Tatgenosse, dessen Verhalten außerordentlich zugerechnet werden würde!

Da die Rechtsprechung bekanntlich bis heute Mord und Totschlag für selbständige Tatbestände hältt, ${ }^{6}$ hatte diese ursprünglich in einem obiter dictum die Möglichkeit einer Mittäterschaft in Zweifel gezogen. Von Mittäterschaft werde nur bei Verwirklichung desselben Grundtatbestandes durch mehrere Täter gesprochen. ${ }^{7}$ In der Literatur wird unterstellt, dass der Bundesgerichtshof in dieser Entscheidung von einem Exklusivitätsverhältnis zwischen Mord und Totschlag ausgegangen sei. ${ }^{8}$ Dies lässt sich jedoch der Entscheidung expressis verbis nicht entnehmen. In dem vom Bundesgerichtshof $\mathrm{zu}$ entscheidenden Fall hat der zuständige Senat diese Zweifel nicht geteilt und eine andere Rechtsauffassung vertreten: Selbst dann, wenn man Mord und Totschlag als selbständige Tatbestände begreife, besage dies nicht notwendig, dass Mörder und Totschläger verschiedene Straftaten im Sinne des § 25 II StGB begehen würden. Zwar würden Mittäter laut § 25 II StGB »die Straftat« gemeinschaftlich begehen. Hierunter sei nicht ein bloßer einheitlicher geschichtlicher Lebensvorgang im Sinne des prozessualen Tatbegriffs zu verstehen, sondern der Straftatbestand des materiellen Rechts. Die Mittäterschaft sei aber nicht akzessorisch ausgestaltet, so dass die rechtliche Beurteilung der einzelnen Tatbeiträge auseinanderfallen könne. Die gemeinsame Straftat setze nicht notwendig die Verletzung (nur) des gleichen Strafgesetzes voraus. Bei der Verletzung unterschiedlicher Strafnormen könne es sich um die gleiche Straftat handeln, wenn die eine in der anderen vollständig enthalten sei und die Täter insoweit (auch) gemeinsam einen identischen Straftatbestand verletzen würden. Werde der von beiden Beteiligten erfüll-

5 BGHSt 36, $231 \mathrm{ff}$.

6 Siehe pars pro toto BGHSt 1, 368, 370; 6, 229, 230; 22, 375, 377.

7 BGHSt 6, 329, 330.

8 So zum Beispiel Kühl, (Fn. 2), § 20 Rn. 102. te Tatbestand bei einem Täter, dem zusätzliche Merkmale zuzurechnen seien, durch einen weitergehenden Straftatbestand verdrängt, so bedeute dies nicht, dass auch bezüglich des gemeinsam erfüllten Delikts verschiedene Straftaten begangen worden seien. Es handele sich vielmehr um einen Fall von Gesetzeskonkurrenz. Demzufolge könne die in beiden Tatbeständen gleichermaßen enthaltene einheitliche Straftat in Mittäterschaft begangen werden. ${ }^{9}$

Dieser Standpunkt entspricht der herrschenden Ansicht in der Literatur. Sofern diese den Mord als Qualifikation des Totschlags begreift, wird für die Möglichkeit von Mittäterschaft ins Feld geführt, dass die mittäterschaftliche Verwirklichung eines Grundtatbestandes nicht dadurch ausgeschlossen werden könne, dass einer der Beteiligten ein Qualifikationsmerkmal verwirkliche. ${ }^{10}$ Verwiesen wird insoweit auch auf $\S 28$ II StGB. ${ }^{11}$ Aber selbst bei Anerkennung der Prämisse, dass es sich um selbständige Tatbestände handelt, wird in der Literatur die Möglichkeit von Mittäterschaft bejaht. Begründet wird dies in Übereinstimmung mit der Rechtsprechung damit, dass die gegenseitige Zurechnung nicht nach Akzessorietätsregeln stattfinde. ${ }^{12}$ Gefordert wird lediglich, dass sich die betreffenden Tatbestände nicht einander ausschließen, dass also zwischen ihnen kein Exklusivitätsverhältnis besteht ${ }^{13}$ bzw. dass der mittäterschaftlich verwirklichte Tatbestand in dem schwereren bzw. minderschweren Tatbestand enthalten ist. ${ }^{14}$ Dasselbe scheint mit der Formulierung gemeint zu sein, dass die rechtliche Beurteilung der einzelnen Tatbeiträge auseinanderfallen könne, solange die Einheit des Ganzen im Rahmen des gemeinschaftlichen Tatentschlusses gewahrt bleibe. ${ }^{15}$

Für denkbar wird es daher zum Beispiel gehalten, dass sämtliche Beteiligten mittäterschaftlich den Tatbestand der Nötigung nach $\S 240$ StGB erfüllen, aber nur einige von ihnen den Tatbestand des Raubes gemäß § 249 StGB. ${ }^{16}$ Verwiesen wird in diesem Zusammenhang auf eine Entschei-

\section{BGHSt 36, 231, $233 \mathrm{f}$.}

10 Roxin, (Fn. 1), § 25 Rn. 236; ohne nähere Erläuterung für die Möglichkeit von Mittäterschaft auch Joecks, Münchener Kommentar, 1. Bd., 2. Aufl., 2011, § 25 Rn. 187; Kindhäuser, Strafrecht Allgemeiner Teil, 5. Aufl., 2011, §40 Rn. 27.

11 Beulke, NStZ 1990, S. 277, 278.

12 Jescheck/Weigend, Lehrbuch des Strafrechts, Allgemeiner Teil, 5. Aufl., 1996, § 63 I 2, S. 676.

13 Hassemer, JuS 1990, S. 148f.; Roxin, (Fn. 1), § 25 Rn. 236; Schünemann, LK, (Fn. 1), § 25 Rn. 169; Kühl, (Fn. 2), § 20 Rn. 102.

14 Roxin, (Fn. 1), § 25 Rn. 236; Kühl, (Fn. 2), § 20 Rn. 102.

15 Jescheck/Weigend, (Fn. 12), § 63 I 2, S. 676.

16 So konsequent Roxin, (Fn. 1), § 25 Rn. 236; Joecks, MK, (Fn. 10), $\S 25$ Rn. 187; Schünemann, LK, (Fn. 1), § 25 Rn. 169. 
dung des Bundesgerichtshofs, in deren zugrundeliegenden Sachverhalt der Angeklagte B nach Anwendung von Gewalt dem Opfer die Brieftasche entwendet hatte. Für fraglich hielt der Bundesgerichtshof die Absicht rechtswidriger Zueignung bezüglich des Angeklagten A, da für diesen aufgrund einer schuldrechtlichen Forderung die Annahme naheliegend gewesen sei, gegenüber dem Opfer einen Anspruch auf Übereignung des entsprechenden Betrags aus jenem Geld zu haben, das dieses bei sich führte. Sofern der Angeklagte A irrtümlich glaubte, Inhaber eines Anspruchs auf Herausgabe eines seiner Forderungen entsprechenden Betrags aus der Geldbörse des Opfers zu sein, kam daher bei diesem Angeklagten nur eine Strafbarkeit wegen Nötigung, möglicherweise in Tateinheit mit Körperverletzung, in Betracht. Bezüglich des Angeklagten B ist hingegen der Bundesgerichtshof von der Verwirklichung des $\S 249$ StGB ausgegangen, sofern er schon vor der Anwendung der Gewalt den Entschluss gefasst hatte, dem Opfer mehr Geld abzunehmen, als dem Angeklagten A zustand. ${ }^{17}$ Der Fall weist die oben geschilderte Problematik auf, sofern man voraussetzt, dass die Gewalt ausschließlich seitens des Angeklagten A ausgeübt wurde. Der mitgeteilte Sachverhalt lässt allerdings diesen Punkt offen.

Ein weiterer Fall ist schon sehr früh vom Reichsgericht entschieden worden. Die Angeklagten $\mathrm{H}$ und W arbeiteten als Küferlehrlinge im Weinkeller des Weinhändlers D. Während des letzten Lehrjahres nahm der Angeklagte H, als sich beide Angeklagte in dem Weinkeller befanden, fünfmal eine Flasche Wein aus dem Lager und steckte sie dem Angeklagten $\mathrm{W} z u$, der diese jeweils an sich nahm und zu Hause austrank. Das Reichsgericht ist von einer gemeinschaftlichen Ausführung eines Diebstahls nach $\S 242$ RStGB ausgegangen, die es bezüglich des Angeklagten $\mathrm{H}$ als Diebstahl und bezüglich des Angeklagten $\mathrm{W}$ privilegierend als Mundraub gemäß § 370 Nr. 5 RStGB eingeordnet hat, weil dieser, ohne dass der Angeklagte $\mathrm{H}$ davon wusste, den Wein mit anderen Hausgenossen austrinken wollte. Das Reichsgericht setzte dabei voraus, dass der Mundraub als lex specialis den Tatbestand des Diebstahls in objektiver und subjektiver Hinsicht vollständig enthält. ${ }^{18}$ Dieser Fall weist die spezifische Problematik auf, wenn man unterstellt, dass der Gewahrsam des Berechtigten schon durch das Zustecken der Flaschen seitens des Angeklagten $\mathrm{H}$ gebrochen und nicht lediglich gelockert wurde.

Was ist für die soeben aufgeführten Fälle kennzeichnend? Das Einverständnis der Tatgenossen erstreckt sich

17 BGH, GA 1968, S. 121.

18 RGSt 12, 8 ff. auf alle verhaltensbezogenen Tatbestandsmerkmale - und zwar sowohl hinsichtlich des Tatbestandes, der von allen Beteiligten verwirklicht wird, als auch hinsichtlich des schwereren oder minderschweren Tatbestandes, der nur von einem Teil der Tatgenossen erfüllt wird. Die Tatumstände, die die Erfüllung des schwereren oder minderschweren Tatbestandes begründen, sind jeweils verhaltensunabhängig. Das heißt, dass sie von vornherein außerhalb des Bereichs der durch § 25 II StGB statuierten außerordentlichen Verhaltenszurechnung liegen. In den hier vorangestellten Beispielen gehören diese Umstände fast ausnahmslos dem subjektiven Tatbestand bzw. wenn man die Mordmerkmale der ersten und dritten Gruppe als spezielle Schuldmerkmale kategorisiert ${ }^{19}$ - der Schuldebene an. Lediglich das Mordmerkmal der Heimtücke zeigt, dass gleichermaßen Tatbestandsmerkmale, die (auch) dem objektiven Tatbestand angehören, einschlägig sind. Eine gute Exemplifizierung dafür bietet auch die Körperverletzung im Amt nach $\S 340$ StGB: Ein Amtsträger und ein Extraneus können mittäterschaftlich die Körperverletzungstatbestände der $\S \S 223 \mathrm{ff}$. StGB verwirklichen, während ausschließlich der Amtsträger nach § 340 StGB bestraft wird. Dem Extraneus verbleibt insoweit lediglich die Rolle eines Gehilfen. ${ }^{20}$

\section{Weitere Erscheinungsformen teilweiser Mittäterschaft?}

In jüngster Zeit ist von Rengier die Problematik in den Fokus der Betrachtung gerückt worden, ob sich die Mittäterschaft auf einen Teil des Tatbestandes beschränken kann, selbst wenn die Beteiligten bezüglich eines weiteren Tatbestandes, der in dem anderen Tatbestand enthalten ist, nicht als Mittäter zu qualifizieren sind. ${ }^{21}$ Rengier bezieht sich auf Fälle, in denen nur einer der Tatgenossen über die vom Tatbestand geforderte innere Absicht verfügt - so zum Beispiel in dem vom Bundesgerichtshof entschiedenen Fall, in dem die Freundin des Angeklagten als Alleintäterin die Geldtasche der Bedienung mit Selbstzueignungsabsicht in ihren Gewahrsam gebracht hatte und der Angeklagte, als dies von der Bedienung bemerkt wurde, diese bedrohte, um seine Freundin im Besitz der Geldasche zu erhalten. Der Bundesgerichtshof hat allerdings in diesem Fall eine Mittäterschaft abgelehnt, weil

19 Siehe dazu Lenckner/Eisele, Sch/Sch, (Fn. 1), Vor §13 Rn. 122. 20 Schünemann, LK, (Fn. 1), §25 Rn. 169.

21 Rengier, Puppe-FS, 2011, S. 849 ff., mit weiteren Beispielen. 
der Angeklagte nicht mit Zueignungsabsicht und dem Willen gehandelt habe, sich den Besitz der entwendeten Geldtasche zu erhalten. Stattdessen hat der Bundesgerichtshof eine Beihilfe des Angeklagten zum schweren räuberischen Diebstahl seiner Freundin angenommen. Der Freundin sei der Einsatz der Nötigungsmittel seitens des Angeklagten zuzurechnen, da sie von vornherein darauf vertraut habe und als eigene Handlung gewollte habe, dass der Angeklagte zu ihrem Schutz eingreifen würde. ${ }^{22}$

Der Umstand, dass der Angeklagte ohne die Absicht, sich im Besitz der Beute zu erhalten, gehandelt hatte, hindert indes nach Auffassung von Rengier die Mittäterschaft nicht. Besondere subjektive Tatbestandsmerkmale wie zum Beispiel in $\S 252$ StGB die Absicht, sich im Besitz der Beute zu erhalten - könnten ohnehin nicht gegenseitig zugerechnet werden. Aufgabe des Tatplans der Tatgenossen sei es allein, den Bereich abzustecken, der durch bewusstes und gewolltes Zusammenwirken gemeinsam verwirklicht werden solle. Wechselseitig zugerechnet würden aufgrund von $\S 25$ II StGB ausschließlich die objektiven Tatbeiträge. ${ }^{23}$ Schon Jakobs hat auf die Möglichkeit aufmerksam gemacht, dass sich das Gemeinsame auf den Handlungsvollzug beschränkt. ${ }^{24}$ Frister spricht insoweit von isolierter Mittäterschaft: Aufgrund der neben dem tatbestandlichen Verhalten zu erfüllenden weiteren Strafbarkeitsvoraussetzungen könne - so seine Auffassung - die Strafbarkeit von gemeinschaftlich handelnden Personen im Ergebnis durchaus unterschiedlich zu beurteilen sein. Wer mit einem anderen zusammen handele, müsse sich dessen Tatbeiträge auch dann zurechnen lassen, wenn in der Person des anderen bestimmte Strafbarkeitsvoraussetzungen nicht verwirklicht seien und dieser deshalb keine oder eine andere Straftat begangen habe. Der Begriff der Mittäterschaft sei deshalb irreführend; die Regelung des $\S 25$ II StGB besage, dass derjenige wie ein Täter zu bestrafen sei, der einen Straftatbestand durch eine zusammen mit einem anderen begangene Handlung verwirklicht habe..$^{25}$ Auch Hoyer vertritt die Ansicht, dass es einer Mittäterschaft nicht entgegenstehe, wenn nur einer der Beteiligten die erforderlichen besonderen Tätermerkmale wie zum Beispiel die Amtsträgereigenschaft oder die vom Tatbestand geforderten Absichten aufweise. ${ }^{26}$

Würde man den Vorgaben der anfangs skizzierten herrschenden Meinung folgen, könnte man immerhin in

22 BGH, StV 1991, S. 349.

23 Rengier, (Fn. 23), S. 949, 950 f.

24 Jakobs, Strafrecht Allgemeiner Teil, 2. Aufl., 1991, 21/45.

25 Frister, Strafrecht Allgemeiner Teil, 5. Aufl., 2011, 25/23.

26 Hoyer, in: Systematischer Kommentar, Bd. 1, 7. Auf., 2000, § 25 Rn. 107. konstruktiver Hinsicht daran denken, dass in dem vollendeten § 252 StGB eine versuchte Nötigung enthalten sei, und darauf die Mittäterschaft gründen, sofern man bezüglich der §§ 240, 22, 23 I StGB von der Erfüllung der Voraussetzungen der Mittäterschaft ausgeht. Die von Rengier ebenfalls zur Diskussion gestellte Abwandlung des Falles, in der die Abrede erfolgt, bevor die Freundin des Angeklagten den Gewahrsam an der Geldtasche erlangt hat, ${ }^{27}$ wäre daher - folgt man der herrschenden Ansicht - noch unproblematischer, da diese sich hier ohne Weiteres darauf berufen könnte, dass die Freundin und der Angeklagte eine Nötigung gemäß $\S 240$ StGB mittäterschaftlich verwirklichen würden. § 249 StGB ist lex specialis zu § 240 StGB. ${ }^{28}$ Die vollendete Nötigung ist im vollendeten Raub enthalten. Es könnten somit die Grundsätze der herrschenden Meinung angewendet und daher eine Mittäterschaft befürwortet werden, wenn man einmal von dem Problem absieht, worin überhaupt der notwendige Tatbeitrag der Freundin des Angeklagten liegen soll.

Die von Rengier in den Raum gestellte Möglichkeit, dass sich die Mittäterschaft auf einen Teil des Tatbestandes beschränkt, könnte man noch weiter auf die Spitze treiben, wie der folgende Fall zeigt: A vereinbart mit B, dass $O$ heftig verprügelt werden soll. Während $B$ die eigentliche Tatbestandshandlung vollzieht, beschränkt sich A plangemäß darauf, die Tatausführung zu ermöglichen und abzusichern. Nachdem B den Tatort verlässt, nutzt A ohne Wissen des B die Wehrlosigkeit des übel zugerichteten $\mathrm{O}$ aus und entwendet dessen Geldbörse. Dass dies sein eigentliches Tatmotiv war, hatte A dem B bei der Tatverabredung verheimlicht. Der Unterschied zu den anderen Fällen liegt darin, dass sich hier bezüglich des $§ 249$ StGB die Vereinbarung zwischen A und B nur auf einen Teil der verhaltensbezogenen Tatbestandsmerkmale richtet. Sie beschränkt sich auf die Ausübung von Gewalt gegen eine Person. Die Wegnahme ist nicht mehr von dem gemeinsamen Tatentschluss erfasst und damit auch nicht die finale Verknüpfung zwischen beiden Tatbestandsmerkmalen seitens A. Gesetzt den Fall, dass das Verhalten des B dem A im Rahmen von § 223 StGB qua § 25 II StGB zugerechnet werden kann, stellt sich das Problem, ob diese Zurechnung auch im Hinblick auf die Strafbarkeit des A nach $\S 249$ StGB Geltung besitzt. Würde man der soeben dargestellten Ansicht von Rengier folgen, käme eine mittäterschaftliche Zurechnung nach § 25 II StGB nicht in Betracht, da es sich bei der Wegnahme um ein verhaltens-

27 Rengier, (Fn. 23), S. 849, 852.

28 BGH, NStZ-RR 2000, S. 206; Vogel, Leipziger Kommentar, Bd. 8, 12. Auf., § 249 Rn. 67. 
bezogenes Tatbestandsmerkmal handelt, auf das sich der gemeinsame Tatentschluss erstrecken muss. Dann aber so scheint es - droht die kaum akzeptable Konsequenz, dass eine Strafbarkeit des A nach $\S 249$ StGB ausgeschlossen ist. Vereinzelt wird in der Literatur vorgeschlagen, auch in diesen Fällen die mittäterschaftliche Zurechnung bejahen, wenn einer der Beteiligten eine vom Tatplan nicht gedeckte zusätzliche Handlung vollzieht und dadurch ausschließlich dieser Beteiligte einen weiteren Tatbestand erfüllt. ${ }^{29}$ Der Bundesgerichtshof ist jedoch kürzlich in einem fast identischen Fall mit keinem Wort auf die Möglichkeit einer teilweisen Mittäterschaft eingegangen, sondern hat in äußerst fragwürdiger Weise eine mittelbare Täterschaft des A aufgrund des Irrtums des B angenommen. ${ }^{30}$

\section{Zur Stichhaltigkeit der Annahme von Mittäterschaft}

Setzt man zunächst beim Rechtsgrund der außerordentlichen Verhaltenszurechnung des § 25 II StGB an, so stellt sich die Frage, ob dieser in den dargestellten Fällen einer mittäterschaftlichen Haftung entgegensteht. Jakobs zufolge handelt es sich bei der Mittäterschaft um eine akzessorische Beteiligungsform..$^{31}$ Er weicht damit offenbar von der oben geschilderten Rechtsauffassung des Bundesgerichtshofs ab. Die in verbindender Arbeitsteilung bestehende Beteiligung im Vorfeld, die die mittäterschaftliche Haftung konstituiert, ist seines Erachtens eine bloße Obliegenheitsverletzung, durch den sich der Betreffende für die spätere Tatausführung »zuständig « macht. Dem potentiellen Mittäter soll das Unrecht der Haupttat zugerechnet werden. Aufgrund dessen wird Jakobs zufolge die Verantwortlichkeit für die Obliegenheitsverletzung zur Verantwortlichkeit für Unrecht und damit zur Schuld. Ein qualitativer Unterschied zur Teilnahme besteht seines Erachtens nicht, sondern lediglich ein Unterschied in der Quantität der Pflichtverletzung. ${ }^{32}$ Würde man diesem Ansatz folgen, wäre die mittäterschaftliche Zurechnung zumindest im letzten Beispielsfall zweifelhaft. Denn die körperliche Gewaltanwendung des B diente nicht dazu, dem A die Wegnahme

29 So Timpe, JZ 1990, S. 97, 98, im Verhältnis von Nötigung und Raub.

30 BGH, NStZ 2013, S. $103 \mathrm{f}$.

31 Jakobs, GA 1996, S. 253, 260; ders., Lampe-FS, 2003, S. 561, 572; ders., Puppe-FS, 2011, S. 547, 558; ebenso Küper, Versuchsbeginn und Mittäterschaft, 1978, S. 60f.; Lesch, ZStW 105 (1993), S. 285, 292.

32 Jakobs, Puppe-FS, (Fn. 31), S. 547, $555 \mathrm{ff}$. zu ermöglichen..$^{33}$ Gegen den Ansatz von Jakobs ist jedoch einzuwenden, dass eine Bestrafung nicht auf eine bloße Obliegenheitsverletzung gestützt werden kann. Damit soll nicht in Abrede gestellt werden, dass die Tatvereinbarung als solche im Vorfeld der eigentlichen Tat verbleibt und insoweit die Mittäterschaft im rein faktischen Sinne akzessorisch ist. Auch würde es gegen das Schuldprinzip verstoßen, einem Beteiligten fremdes personales Unrecht eines anderen Beteiligten zuzurechnen. Nur für eigenes personales Unrecht kann man strafrechtlich haftbar gemacht werden.

Nach hier vertretener Auffassung beruht die außerordentliche Verhaltenszurechnung der Mittäterschaft darauf, dass jeder der Mittäter nicht nur für sich selbst, sondern ebenso für seine Tatgenossen handelt, indem er sich wie ein Stellvertreter ihren Willen unterordnet und sich ihnen dienstbar macht. Für diese Willensordnung sind die anderen Mittäter ihrerseits aufgrund der gemeinsamen Tatvereinbarung verantwortlich. Denn durch diese ermächtigen sie den betreffenden Mittäter dazu, seinen Tatbeitrag auch in ihrem Namen zu erbringen. ${ }^{34}$ Die Mittäter sind also bezüglich des von dem Tatgenossen vollzogenen Tatbeitrags mittelbare Täter, bezüglich des eigenen Tatbeitrags unmittelbare Täter. ${ }^{35}$ Der Zurechnungsgrund liegt also in der wechselseitigen Repräsentation, aufgrund derer die einzelnen Mittäter voneinander als Tatgenossen ununterscheidbar sind. ${ }^{36}$ Die Rechtsprechung hält es für maßgebend, ob der potentielle Mittäter nicht nur fremdes Tun gefördert, sondern den eigenen Tatbeitrag derart in eine gemeinschaftliche Tat eingefügt hat, dass sein Beitrag als Teil der Tätigkeit des anderen und umgekehrt dessen Tun als Ergänzung seines Tatanteils erscheint. ${ }^{37}$ Mit dieser Formulierung wird allerdings nichts anderes zum Ausdruck gebracht als die soeben umschriebene ratio der Verhaltenszurechnung. Denn der Tatbeitrag des einen Mittäters ist nur deswegen Teil der Tätigkeit des anderen Mittäters, weil dieser seinen Tatgenossen aufgrund der ausdrücklichen oder konkludenten Tatvereinbarung dazu autorisiert hat, den von ihm zu leistenden Tatbeitrag auch für ihn zu erbringen.

33 Vgl. die Definition der Gewalt bei Küper, Strafrecht Besonderer Teil, 7. Aufl., 2008, S. 179.

34 Haas, Die Theorie der Tatherrschaft und ihre Grundlagen, 2008, S. 113.

35 Ebenso schon in konstruktiver Hinsicht RGSt 58, 279; 66, 236, 240; $71,23,24$.

36 So Kindhäuser, Hollerbach-FS, 2001, 635ff.; ihm folgend Haas, (Fn. 34), S. 112.

37 Pars pro toto BGHSt 6, 248, 249; 8, 393, 396; 37, 289, 291. 
Die Tatherrschaftslehre kann hingegen die Mittäterschaft nicht erklären. Grundprämisse der Tatherrschaftslehre ist es laut Roxin, dass das vollverantwortliche Verhalten des Vordermanns die Herrschaft des Hintermanns über die Tat ausschließt. Aus diesem Grund soll sich die Mitherrschaft der Mittäter über die Gesamttat gerade nicht auf die Beherrschung der Komplizen und ihrer Tatbeiträge stützen können. ${ }^{38}$ Wie aber soll es möglich sein, dass der einzelne Mittäter die Gesamttat beherrscht, ohne alle ihre Teile zu beherrschen? $\mathrm{Zu}$ beachten ist, dass es Funktion des § 25 II StGB ist, den Beteiligten als Täter für die Tatbeiträge strafrechtlich zur Verantwortung zu ziehen, die er nicht schon selbst in persona vollzogen hat. Die Tatherrschaftslehre gründet die Mitherrschaft über die Gesamttat auf die positive und negative funktionelle Tatherrschaft. ${ }^{39}$ Die positive funktionelle Tatherrschaft soll die Fähigkeit bezeichnen, die Tat durch das Erbringen des eigenen Tatbeitrags ablaufen lassen zu können. Diesbezüglich ergibt sich allerdings die Schwierigkeit, dass diese Fähigkeit aufgrund der fehlenden Beherrschung der Tatbeiträge der Tatgenossen auf den eigenen Tatbeitrag begrenzt ist. Die negative funktionelle Tatherrschaft bezeichnet hingegen die Fähigkeit, die Tat durch die Verweigerung der Erbringung des eigenen Tatbeitrags verhindern oder hemmen zu können. Diesbezüglich sieht sich jedoch die Lehre mit dem Problem konfrontiert, dass auch der Teilnehmer über eine derartige negative Hemmungsmacht verfügen kann. Die Tatherrschaftslehre vermag es also nicht, Täterschaft und Teilnahme qualitativ vollständig voneinander abzugrenzen. ${ }^{40}$ Sie übersieht zudem, dass die Konzeption des $\S 25$ II StGB als Zurechnungsvorschrift zwingend dazu führt, dass jeder der Mittäter als mittelbarer Täter bezüglich der Tatbeiträge der anderen behandelt wird. Die Tatherrschaftslehre stellt aber genau dies in Abrede, ${ }^{41}$ obwohl Roxin früher - aufgrund seiner Prämissen in sich völlig schlüssig - die Mittäterschaft nicht als Zurechnungsfigur aufgefasst hatte. ${ }^{42}$

Folgt man indes der hier vertretenen Lehre, handelt es sich entgegen der Ansicht von Jakobs bei der Mittäterschaft um keine akzessorische Beteiligungsform im normati-

38 Roxin, Täterschaft und Tatherrschaft, 8. Aufl., 2006, S. 276f., 287, 290, 292, 294; ebenso Rudolphi, Bockelmann-FS, 1979, S. 369, 373f., 383.

39 Vgl. Maurach, Deutsches Strafrecht Allgemeiner Teil, 2. Aufl., 1958, S. 517; Roxin, (Fn. 38), S. 276f., 287, 294, 311; Zieschang, ZStW 107 (1995), S. 361, 373.

40 Siehe Haas, (Fn. 34), S. 32ff.

41 Bloy, Die Beteiligungsform als Zurechnungstypus im Strafrecht, 1985, S. 266; Kühl, (Fn. 2), § 20 Rn. 98ff.; nunmehr auch Roxin, Odersky-FS, 1996, S. 494.

42 Roxin, (Fn. 38), S. 292. ven Sinne. Der Zurechnungsgrund der Mittäterschaft setzt - anders als bei der eigentlichen Teilnahme - nicht voraus, dass der Komplize, dessen Verhalten dem anderen Mittäter zugerechnet werden soll, in seiner Person als Täter das Unrecht verwirklicht, für das sich der Mittäter als Zurechnungsadressat verantworten soll - im letzten Beispielsfall also § 249 StGB. Es kommt ausschließlich darauf an, dass das zugerechnete Verhalten in seiner Person als Zurechnungsadressaten Unrecht verwirklicht. Nicht die Tatbestandsverwirklichung bzw. das tatbestandliche Unrecht wird zugerechnet, sondern lediglich das Verhalten des Tatgenossen. ${ }^{43}$ Verwirklicht das zugerechnete Verhalten in der Person des Zurechnungsadressaten Unrecht, so ist auch die Einverständniserklärung des betreffenden Mittäters als solche - unter der Präsumtion ihrer nicht erfolgenden Revokation - Unrecht. Denn es gilt: Ist es rechtswidrig, dass eine bestimmte Person ein bestimmtes Verhalten vollzieht, dann ist es auch grundsätzlich rechtswidrig, dass diese Person einen anderen ermächtigt, dieses Verhalten stellvertretend für sie zu vollziehen.

Selbst im letzten Beispielsfall scheint daher einer Strafbarkeit des A als Mittäter nichts im Wege zu stehen. Ist die Zurechnung nicht im normativen Sinne akzessorisch, weil nur das Verhalten als solches außerordentlich zugerechnet wird und der Zurechnungsgrund nicht voraussetzt, dass das Verhalten des Vordermanns ihm gegenüber rechtswidrig ist, wäre es widersprüchlich, die außerordentliche Zurechnung des Verhaltens des B gegenüber A ihrem Rechtsgrund nach nur relativ auf einen bestimmen Tatbestand anzuerkennen - also etwa zu formulieren, dass die außerordentliche Verhaltenszurechnung nur im Rahmen des $\S 223$ StGB gelte, nicht aber im Rahmen des § 249 StGB. Entscheidend wäre hinsichtlich des Raubes allein, dass A aufgrund der Verhaltenszurechnung in seiner Person alle Tatbestandsmerkmale des § 249 StGB verwirklichen würde.

So zutreffend diese Erwägungen unter ausschließlicher Berücksichtigung des Rechtsgrundes der Verhaltenszurechnung sind, so fragwürdig sind sie - was auch seitens des Verfassers verkannt worden ist $\mathrm{t}^{44}$ - unter Berücksichtigung des Wortlauts des § 25 II StGB, dem zufolge Voraussetzung der Mittäterschaft die gemeinschaftliche Begehung der Straftat ist. So verneint ein Teil der Literatur im oben geschilderten Fall des räuberischen Diebstahls die Möglichkeit einer Mittäterschaft. Als Voraussetzung

43 Dass die Mittäterschaft keine akzessorische Beteiligungsform im normativen Sinne ist, ist von Bedeutung, wenn die verletzte Rechtsposition an einen bestimmten personalen Status gebunden ist, den nicht alle Beteiligten aufweisen.

44 Haas, (Fn. 34), S. 113. 
einer Zurechnung nach $\S 25$ II StGB wird bei § 252 StGB gefordert, dass der Beteiligte, dessen Verhalten zugerechnet werden soll, mit der Absicht, sich im Besitz der Beute zu erhalten, gehandelt haben müsse. ${ }^{45} \mathrm{Ganz}$ generell wird verlangt, dass nicht nur Täterqualifikationen, sondern auch täterschaftsbegründende Absichten bei allen Mitwirkenden gegeben sein müssten, damit Mittäterschaft angenommen werden könne. ${ }^{46}$ Begründet wird diese Auffassung damit, dass die Mittäterschaft konstruktiv von der wechselseitigen Zurechnung lebe. Nur einen Täter könne es daher bei der Mittäterschaft nicht geben. ${ }^{47}$

Sofern mit der Ansicht, dass die Mittäterschaft konstruktiv von der wechselseitigen Zurechnung lebt, behauptet werden soll, dass der Rechtsgrund der außerordentlichen Verhaltenszurechnung ihre Wechselseitigkeit voraussetzt, wäre ihr nicht zu folgen. Hingegen wäre ihr beizupflichten, wenn sie lediglich darauf aufmerksam machen möchte, dass die in $\S 25$ II StGB normierte Mittäterschaft den Spezialfall der reziproken außerordentlichen Verhaltenszurechnung regelt, bei der die Tatgenossen dieselbe materiell-rechtliche Tat begehen, und dass die Tatbestandsbezogenheit der Mittäterschaft Konsequenz dieses Umstands ist. Es ist trivial: Täter ist man immer nur im Hinblick auf eine bestimmte Tat. Die Feststellung, dass eine Person Mittäter ist, setzt daher die Feststellung voraus, dass es mindestens einen zweiten Täter derselben Tat gibt, bei dem ebenfalls die Mechanismen der außerordentlichen Verhaltenzurechnung der Mittäterschaft eingreifen. Es wäre widersprüchlich, einerseits die Tatbestandsbezogenheit der Mittäterschaft zuzugestehen, andererseits aber von einer gemeinsamen Tat bei Verwirklichung verschiedener Strafgesetze auch dann zu sprechen, wenn es sich um selbständige Tatbestände handelt. ${ }^{48}$ Die Tatbestandsbezogenheit der Mittäterschaft spricht daher - anders als in dem anfänglich geschilderten einfach $\mathrm{zu}$ lösenden Ausgangsfall - in den hier problematisierten Beispielsfällen gerade gegen eine Anwendung von § 25 II StGB.

Insoweit läuft auch die oben wiedergegebene, im Hinblick auf den Rechtsgrund der mittäterschaftlichen Verhaltenszurechnung plausible Anmerkung von Rengier, dass die subjektiven Tatbestandsmerkmale ohnehin nicht wechselseitig zugerechnet werden würden und daher der gemeinsame Tatplan sich auch nicht auf diese erstrecken müsse, ins Leere. Bedenklich ist des Weiteren die oben wiedergegebene Ansicht von Frister, dass der Begriff der

45 Hillenkamp, JuS 2003, S. 157, 160; Dehm-Niemann/Weber, JA 2009, S. 868, 870 .

46 Gropp, Strafrecht Allgemeiner Teil, 3. Aufl., 2005, § 10 Rn. 86. 47 Dehm-Niemann, JuS 2008, S. 589, 591.

48 So jedoch zum Beispiel Timpe, JZ 1990, S. 97, 98.
Mittäterschaft irreführend sei und dass die Regelung des $\S 25$ II StGB eigentlich besage, dass derjenige wie ein Täter zu bestrafen sei, der einen Straftatbestand durch eine zusammen mit einem anderen begangenen Handlung verwirklicht. Diese Rekonstruktion weitet den Anwendungsbereich des § 25 II StGB über seinen Wortlaut hinaus aus und ist daher im Hinblick auf Art. 103 II GG zweifelhaft, der - zumindest nach Auffassung der Rechtsprechung, ${ }^{49}$ aber auch von weiten Teilen der Literatur ${ }^{50}$ - auch im Allgemeinen Teil des StGB Geltung beansprucht.

Die anfänglich referierte Rechtfertigung des Bundesgerichtshofs für die Anerkennung der Möglichkeit einer Mittäterschaft bei Mord und Totschlag, wenn sämtliche Tatgenossen einen Totschlag begehen, aber nur beim $\mathrm{Zu}$ rechnungsadressaten die Voraussetzungen des Mordes erfüllt sind, blendet diese Problematik aus. Sofern der Bundesgerichtshof entgegen seiner früheren Auffassung darauf verweist, dass auch bei der Verletzung unterschiedlicher Strafnormen dann gemeinschaftlich eine Straftat begangen werde, wenn die eine Strafnorm in der anderen vollständig enthalten sei, so dass bezüglich des gemeinsam erfüllten Delikts dieselbe Straftat begangen worden sei, übersieht er, dass es sich gerade im Hinblick auf den Straftatbestand, den allein der Zurechnungsadressat verwirklicht, um dieselbe Straftat handeln muss. Dafür ist es jedoch nicht ausreichend, dass der eine Straftatbestand in dem anderen enthalten ist! Voraussetzung dafür wäre vielmehr - im Einklang mit der früheren Rechtsprechung -, dass es sich bei dem spezielleren Delikt um eine Qualifikation oder Privilegierung des Grundtatbestandes handelt. ${ }^{51}$ Unklar ist ferner die Auffassung des Bundesgerichtshofs, dass die in beiden Tatbeständen - in $\S 211$ StGB und $\S 212$ StGB - gleichermaßen enthaltene einheitliche Straftat in Mittäterschaft begangen werden könne. Eine dritte, in beiden Tatbeständen enthaltene Straftat existiert nicht. ${ }^{52}$

Im Ergebnis ist daher der Ansicht von Teilen der Literatur beizupflichten, die aus der Teilbarkeit der Mittäterschaft bezüglich des zentralen Problems dieses Aufsatzes folgert, dass die Mittäterschaft für jeden Tatbestand gesondert festgestellt werden müsse. Die mittäterschaftliche $\mathrm{Zu}$ -

49 Vgl. BVerfGE 96, 68, 97; BGHSt 35, 347, 350; 42, $158 \mathrm{ff}$.

50 Pars pro toto Dannecker, LK, (Fn. 1), §1 Rn. $84 \mathrm{ff}$.

51 Die von Küper, JZ 1990, S. 862, 868; ders., JZ 2006, S. 1157, 1158, zu Recht angerissene Frage, worin der materiell-rechtliche Unterschied zwischen einer Qualifikation und einem selbständigen Tatbestand trotz derselben logischen Struktur der Spezialität liegt, kann hier nicht beantwortet werden.

52 Ablehnend im Ergebnis auch Schneider, Münchener Kommentar, 4. Bd., 2. Aufl., 2012, § 211, Rn. 257, allerdings mit zirkulärer Argumentation. 
rechnung nach $\S 25$ II StGB soll demnach beim räuberischen Diebstahl nicht darauf gestützt werden können, dass die (versuchte) Nötigung mittäterschaftlich begangen wurde. ${ }^{53}$ Dies gilt auch für alle anderen Fälle, in denen ein Tatbestand in einem anderem selbständigen Tatbestand enthalten ist. Die Reziprozität der außerordentlichen Verhaltenszurechnung kommt damit in ihrer Auswirkung der normativen Akzessorietät nahe.

\section{Die Lösung der Problematik}

Die vorstehenden Ausführungen geben allerdings schon einen Hinweis auf den zu beschreitenden Weg, um einer kaum nachvollziehbaren Strafbarkeitslücke zu entgehen. Wenn sich die Mittäterschaft als Kombination unmittelbarer und mittelbarer Täterschaft erweist, dann bietet es sich an, bei einer einseitigen außerordentlichen Verhaltenszurechnung direkt auf $\S 25$ I 2. Var StGB zurückzugreifen. Voraussetzung für die Möglichkeit eines Rückgriffs auf $\S 25$ I 2. Var StGB ist allerdings, dass die Beauftragung eines anderen, für den Beauftragenden eine Straftat zu vollziehen, auch de lege lata als mittelbare Täterschaft ausgewiesen werden kann. Ebenso wie bei der Mittäterschaft rechtfertigt sich die Rechtsfigur aus der Repräsentation des Vertretenen durch den Vertreter - bloß mit dem Unterschied, dass die Repräsentation nicht wechselseitig erfolgt. Das Handeln für einen anderen ist ein allgemein anerkannter Zurechnungsgrund, der auch in anderen Rechtsgebieten - wie zum Beispiel im Recht der Stellvertretung oder im Bereicherungsrecht - Anwendung findet. ${ }^{54}$ Dennoch hat man an dieser Lehre kritisiert, dass sie einen sehr weiten Begriff mittelbarer Täterschaft konstituiere. ${ }^{55}$ Warum diese Weite dogmatisch auf tönernen Füßen steht, wird allerdings nicht weiter begründet. Analysiert man genau, ist der Begriff nicht weiter als derjenige der Mittäterschaft, wenn man berücksichtigt, dass dem Mittäter jeweils die Tatbeiträge außerordentlich zugerechnet werden sollen, die er nicht selbst vollzogen hat, und dass für die Legitimation dieser Verhaltenszurechnung die eigenen Tatbeiträge des Zurechnungsadressaten rechtlich vollkommen unerheblich sind. Dass die eigenen Tatbeiträge des Zurechnungsadressaten für die Legitimation der Verhaltenszurechnung unerheblich sind, kann man sich an dem Fall vor Augen führen, indem der Mittäter erst dann seinen Tatbeitrag erbringt, nachdem die Tatgenossen ihre

53 Dehm-Niemann, JuS 2008, S. 589, 591; Dehm-Niemann/Weber, JA 2009, S. 868, 870 .

54 Siehe Haas, (Fn. 34), S. 85.

55 So Jakobs, Puppe-FS, (Fn. 31), S. 547, 555, Fn. 45.
Mitwirkungsakte schon vollzogen haben. ${ }^{56}$ Der Umfang der zugesagten Tatbeteiligung ist daher lediglich auf prozessrechtlicher Ebene ein Indiz für die wechselseitige Repräsentation, also dafür, dass die anderen Mittäter auch für ihn handeln. ${ }^{57}$

Historisch kann die dogmatische Formel, dass derjenige, der einen anderen beauftragt, eine Tat zu begehen, so behandelt werden könne, als habe er die Tat selbst begangen, bis Ulpian zurückverfolgt werden. Im 19. Jahrhundert wurde das Mandat als intellektuelle Urheberschaft eingeordnet, die der physischen Urheberschaft gleichstehen sollte. Daran änderte auch nichts der Umstand, dass sich der Begriff des Anstifters einbürgerte, wenngleich die klare Abgrenzung zum consilium, das sich auf das bloße Anraten der Deliktsbegehung bezog, vereinzelt verloren zu gehen drohte. Das preußische Strafgesetzbuch von 1851 ließ unter dem Einfluss des französischen Rechts eine gewisse Hinwendung zum formal-objektiven Täterbegriff erkennen, die jedoch von der strafrechtsdogmatischen Literatur nicht aufgegriffen wurde. Das Reichsstrafgesetzbuch stellte allerdings in $\S 48$ RStGB, da die Norm alle Mittel der Bestimmung des Haupttäters erfasste, das mandatum mit dem consilium gleich und gestaltete es als akzessorische Teilnahmeform im heutigen Sinne aus. Die in den Motiven zum Strafgesetzbuch für den Norddeutschen Bund zu findende Erklärung, dass die Tat des Angestifteten die Tat des Anstifters selbst sei, knüpfte freilich an die oben wiedergegebene tradierte gemeinrechtliche Formel an, die jedoch das mandatum gerade - wie soeben dargelegt - als Urheberschaft konzeptualisiert hatte..$^{58}$ Die unter der Geltung des Reichsstrafgesetzbuchs praeter legem entwickelte mittelbare Täterschaft setzte nicht einfach die Lehre von der intellektuellen Urheberschaft fort. Denn sie erfasste Konstellationen, die die Doktrin des 19. Jahrhunderts ganz überwiegend der physischen Urheberschaft zugeordnet hätte, und diente in Anbetracht der strikt ausgestalteten Akzessorietät der Teilnahme auch der Schließung von Strafbarkeitslücken in Fällen, in denen es beim Vordermann an einer Strafbarkeitsvoraussetzung fehlte. Mehrmals hat jedoch das Reichsgericht im Sinne der subjektiven Theorie eine mittelbare Täterschaft auch bei einer Beauftragung des Ausführenden angenommen, wenn dem Vordermann der Täterwillen fehlte. ${ }^{59}$

Die merkwürdige Zwitterstellung der Anstiftung, die schon seinerzeit von Nagler und Binding heftig kritisiert

56 Ausführlich zu dieser Problematik Haas, (Fn. 34), S. $115 \mathrm{ff}$. 57 Haas, Matt/Renzikowski, Strafgesetzbuch, 2013, § 25 Rn. 82f. 58 Siehe die umfassende Darstellung der Dogmengeschichte bei Haas, (Fn. 34), S. 86ff. 59 So zum Beispiel RGSt 31, 80, 82; 41, 62, 64; 44, 69, 72. 
wurde, ${ }^{60}$ kennzeichnet auch den heutigen $\S 26$ StGB. So wurde in der Begründung zum Entwurf eines Strafgesetzbuchs aus dem Jahre 1962 ausgeführt, dass der Anstifter das Ziel verfolge, eine fremde Tat auszulösen. ${ }^{61}$ Dies gilt jedoch nur für das consilium. Mit der Formulierung, dass der Anstifter wie der Täter bestraft werde und insofern so behandelt werde, als ob er die Tat selbst begangen habe, ${ }^{62}$ wird hingegen an die auf einer Verhaltenszurechnung beruhende Tradition der intellektuellen Urheberschaft angeknüpft, die das mandatum voraussetzt. Für den Auftraggeber ist jedoch die Tat des Ausführenden, der sich dem Willen seines Auftraggebers unterordnet, seine eigene und keine fremde. Die Regelungskonzeption des $\S 26 \mathrm{StGB}$ ist daher in sich widersprüchlich. Schon aufgrund ihrer Widersprüchlichkeit kann daher de lege lata aus der Vorschrift kein Argument gegen die Rechtsfigur einer mittelbaren Täterschaft kraft Beauftragung abgeleitet werden: Ex falso sequitur quodlibet.

Die Formulierung des die mittelbare Täterschaft umschreibenden § 25 I 2. Var StGB greift indes auf jene Formel zurück, die jahrhundertelang die intellektuelle Urheberschaft kennzeichnete - das Begehen der Tat durch einen anderen. Der Wortlaut des $\S 25$ I 2. Var StGB schließt daher die Anwendung der Norm auf die Fälle des mandatum nicht aus. Er legt sie vielmehr nahe. Der Gesetzgeber des 2. Strafrechtsreformgesetzes hat die vorgefundene Dogmatik und Rechtsprechung des Reichsgerichts nicht einfach übernommen. In den Beratungen der Großen Strafrechtskommission war umstritten, ob die Voraussetzungen der mittelbaren Täterschaft gesetzlich umschrieben und in das Strafgesetzbuch die Formel aufgenommen werden sollte, dass als Täter auch bestraft werde, wer vorsätzlich die Straftat durch einen anderen ausführt, der ohne Vorsatz oder trotz Vorsatzes schuldlos handelt oder bei dem nicht die besonderen Eigenschaften, Verhältnisse oder Umstände oder besonderen Absichten vorliegen, welche die Strafbarkeit begründen. Mit dieser Kennzeichnung sollte unter anderem gerade die Möglichkeit ausgeschlossen werden, denjenigen als Täter zu behandeln, der sich mit animus auctoris eines vollverantwortlichen Tatmittlers bedient. Teilweise wurde jedoch auch gegen eine gesetzliche Festlegung seiner Voraussetzungen plädiert. Die Rechtsfrage, ob in diesem konkreten Fall eine mittelbare Täterschaft angenommen werden kann, sollte offenbleiben. ${ }^{63}$

60 Nagler, Die Teilnahme am Sonderverbrechen, 1903, S. 143 ff.; Binding, GS 71 (1908), S. 1, 3, 14; ders., GS 78 (1911), S. 34, 41, 46.

61 BT-DrS IV/650, S. 150.

62 BT-DrS IV/650, S. 150.

63 Niederschriften über die Sitzungen der Großen Strafrechtskommission, 2. Bd., 1958, S. 72ff., S. $115 \mathrm{ff}$.
In dem Entwurf eines Strafgesetzbuchs von 1962, dessen hier interessierende Beteiligungsregeln in dem Bericht des Sonderausschusses kommentarlos übernommen ${ }^{64}$ und durch das 2. Strafrechtsreformgesetz umgesetzt wurden, wurde schließlich angesichts der Vielgestaltigkeit der Formen mittelbarer Täterschaft darauf verzichtet, die Rechtsfigur »in Einzelheiten zu umschreiben«. Als weiterer Grund für diesen Verzicht wurde ausdrücklich angeführt, dass die rechtliche Beurteilung des vollverantwortlichen Tatmittlers noch der Klärung durch die Wissenschaft bedürfe und der Rechtsentwicklung insoweit nicht vorgegriffen werden solle! ${ }^{65}$ Auch im Sonderausschuss wurde auf eine lückenlose Definition der mittelbaren Täterschaft verzichtet, um die Fortentwicklung des Rechts in diesem Bereich nicht zu behindern. ${ }^{66}$ So wendet die Judikatur § 25 I 2. Var StGB auch dann an, wenn der Tatmittler kein Strafbarkeitsdefizit aufweist - wie zum Beispiel unter Umständen beim vermeidbaren Verbotsirrtum des Vordermanns oder bei der mittelbaren Täterschaft kraft Organisationsherrschaft. ${ }^{67}$ Aus demselben Grund wurde auch die Tatherrschaftslehre nicht gesetzlich verankert, wenngleich man ihr Raum geben wollte. ${ }^{68}$ Dies ist deswegen wichtig $\mathrm{zu}$ erwähnen, weil die Einordnung des mandatum als mittelbare Täterschaft mit der Tatherrschaftslehre nicht vereinbar wäre, da die volle Verantwortlichkeit des Tatmittlers die Tatherrschaft des Hintermanns dieser Theorie zufolge ausschließt. Es wurde allerdings schon oben gezeigt, dass diese Grundprämisse der Tatherrschaftslehre nicht tragfähig ist.

Umgekehrt wird die hier vertretene Beteiligungslehre durch die Entwurfsbegründung nicht ausgeschlossen. Abgelehnt wurde nur die subjektive Theorie, insofern diese eine objektive Unterscheidbarkeit von Täterschaft und Teilnahme aufgrund der postulierten Gleichwertigkeit aller Bedingungen in Abrede stellte und die Abgrenzung beider Beteiligungsformen auf den inneren Willen des jeweiligen Beteiligten stützte. ${ }^{69}$ Dieser Ansatz entspricht jedoch nicht der hier vertretenen Position: Erstens erteilt sie der Äquivalenztheorie der Kausalität eine Absage, indem sie davon ausgeht, dass der Hintermann durch seine Einwirkung auf den Vordermann den Taterfolg nicht unmittelbar verursacht und daher durch sein eigenes Verhalten den Tatbestand nicht eigenhändig verwirklicht. Aus diesem Grund bedarf es gerade einer außerordentlichen Ver-

64 BT-DrS V/4095, S. 14.

65 BT-DrS IV/650, S. 149.

66 Sonderausschuss, Protokolle V, S. 1648, 1824.

67 BGHSt 35, 347, 353f.; 40, 218, 236.

68 BT-DrS IV/650, S. $147 \mathrm{f}$.

69 BT-DrS IV/650, S. 147; Sonderausschuss, Protokolle V, S. $1821 \mathrm{ff}$. 
haltenszurechnung. ${ }^{70}$ Und zweitens ist das Mandat kein lediglich interner Wille im Sinne eines animus auctoris, die Tat des Vordermanns als eigene zu wollen. Die Beauftragung ist ein aufklärbarer Sachverhalt der Außenwelt. Die hier vertretene Lehre zwingt auch nicht dazu, denjenigen, der für einen anderen eine Tat begeht, lediglich als Gehilfen einzustufen. Derjenige, der in eigener Person einen Tatbestand erfüllt, ist im Einklang mit der formalobjektiven Theorie immer Täter. ${ }^{71}$ Der berechtigte Kern der subjektiven Theorie liegt ausschließlich in der Rechtsfigur des Mandats.

Die Anerkennung einer mittelbaren Täterschaft kraft Mandats würde die Unstimmigkeiten beseitigen, durch die die bisherige höchstrichterliche Rechtsprechung belastet wird. Einerseits übernimmt der Bundesgerichtshof die Lehre von der mittelbaren Täterschaft kraft Organisationsherrschaft und verlangt, dass der Hintermann bestimmte Rahmenbedingungen ausnutzen muss, innerhalb derer sein Tatbeitrag regelhafte Abläufe auslöst. Derartige Rahmenbedingungen mit regelhaften Abläufen sollen insbesondere bei staatlichen, unternehmerischen und geschäftsähnlichen Organisationsstrukturen und Befehlshierarchien in Betracht kommen. ${ }^{72}$ Andererseits finden sich zunehmend Entscheidungen, in denen der Verdacht besteht, dass die Rechtsprechung formelhaft diese materiell-rechtlichen Vorgaben fortführt, ohne allerdings die forensische Überprüfung einzufordern, dass überhaupt tatsächlich hinreichende Organisationsstrukturen vorhanden gewesen sind. ${ }^{73}$ Man fragt sich daher, warum es dann überhaupt noch der Einbettung der Anweisung oder Aufforderung in eine Organisationsstruktur bedarf. Dass die Rechtsfigur der mittelbaren Täterschaft kraft organisatorischer Machtapparate ohnehin dogmatisch nicht tragfähig ist, soll an dieser Stelle nicht noch einmal dargelegt werden. ${ }^{74}$

Sodann finden sich Entscheidungen, in denen der Bundesgerichtshof auf die Mittäterschaft ausweicht und diese in Fällen anwendet, in denen das Verhalten des Betreffenden lediglich darin besteht, eine andere Person zu beauftragen, eine bestimmte Tat zu begehen, oder sich mit der Tatbegehung einer anderen Person einverstanden

70 Siehe Haas, (Fn. 34), S. $58 \mathrm{ff}$.

71 Haas, Matt/Renzikowski-StGB, (Fn. 57), Vor $\S 25$ Rn. 5.

72 Pars pro toto BGHSt 40, 218, 236; 45, 270, $296 \mathrm{ff}$.

73 Siehe zum Beispiel BGHSt 40, 257, 267 f.; BGH, NStZ 1997, S. 544f.; NJW 1998, S. 767 ff.; JR 1999, S. 105; wistra 2001, S. 386 ff.; NJW 2004, S. $296 \mathrm{ff}$.

74 Grundlegende Kritik bei Renzikowski, Restriktiver Täterbegriff und fahrlässige Beteiligung, 1997, S. 89; Rotsch, ZStW 112 (2000), S. 518f.; siehe ferner auch Haas, (Fn. 34), S. $107 \mathrm{ff}$. zu erklären. ${ }^{75}$ Die Vereinbarung einer Tat kann aber schon aus logischen Gründen kein hinreichender Tatbeitrag im Sinne des § 25 II StGB sein, da Gegenstand der Tatvereinbarung die gemeinsame Tatausführung ist. ${ }^{76}$ Der Rückgriff auf $\S 25$ II StGB hat überdies die Konsequenz, dass die spezifischen Anforderungen, die die Rechtsprechung bei der mittelbaren Täterschaft zumindest offiziell stellt, unterlaufen werden. Schließlich beschreitet die Rechtsprechung noch den dritten Weg, ohne jede nähere dogmatische Erklärung von einer Täterschaft auszugehen - wie zum Beispiel in dem oben aufgeführten Fall des räuberischen Diebstahls, in dem sich der Bundesgerichtshof in fast schon apokrypher Weise auf die subjektive Theorie berufen hat.

Würde man dem hier unterbreiteten Vorschlag folgen, wäre es allerdings unschlüssig, mit einer im Zunehmen begriffenen Auffassung in der Literatur, die Anstiftung auf Fälle zu begrenzen, in denen der Anstifter den Angestifteten dazu verpflichtet, die Tat für ihn zu begehen. ${ }^{77}$ Diese sich auf die Strafdrohung des $\S 26$ StGB stützende Lehre stellt jedoch ohnehin eine Gesetzeskorrektur dar. Der Gesetzgeber hat die tätergleiche Bestrafung des Anstifters damit gerechtfertigt, dass der Anstifter den entscheidenden Anstoß zur Tat gibt. ${ }^{78}$ So lässt die Rechtsprechung grundsätzlich jede Art und Weise der Herbeiführung des Tatentschlusses für die Anstiftung ausreichen. ${ }^{79}$ De lege lata ist $\S 26$ StGB somit auch bei einem bloßen Ratschlag, die Tat zu begehen, anzuwenden. Bei einem bloßen Anraten der Deliktsbegehung greifen die Voraussetzungen einer außerordentlichen Verhaltenszurechnung nicht, und es bedarf - ebenso wie bei der Beihilfe, dem auxilium im Sinne der gemeinrechtlichen Lehre - eines anderen Modus der Unrechtsbegründung. ${ }^{80}$ De lege ferenda ist jedoch eine $\S 27$ II StGB entsprechende obligatorische Strafmilderung einzufordern.

\section{Ergebnis und Ausblick}

Die Untersuchung gelangt damit zu folgenden Ergebnissen: Wenn die Verhaltenszurechnung der Mittäterschaft

75 BGHSt 37, 289, 292; BGH, StV 1986, S. 384; NStZ 1989, S. 574; 1995, S. 122.

76 Haas, Matt/Renzikowski-StGB, (Fn. 57), § 25 Rn. 81.

77 So mit unterschiedlichen Formulierungen Puppe, GA 1985, S. 111ff.; ebenso Jakobs, (Fn. 24), 22/22; Joecks, MK, (Fn. 10), § 26 Rn. 16 ff.; Hoyer, SK (Fn. 26), § 26 Rn. 13.

78 Sonderausschuss, Protokolle V, S. 1828; BT-Dr5/4095, S. 12.

79 BGHSt 9, 379; 45, 373, 374f.; BGH, NStZ 1994, 29, 30; 2000, S. 421f.; 2001, S. 41, 42; StV 2001, S. 406; NStZ 2008, S. 42.

80 Siehe Haas, (Fn. 34), $130 \mathrm{ff}$. 
dazu führen würde, dass der Zurechnungsadressat einen Tatbestand erfüllt, den der Tatgenosse, dessen Verhalten dem anderen außerordentlich zugerechnet werden soll, nicht verwirklicht, greift § 25 II StGB nur dann ein, wenn der Tatbestand, den der Zurechnungsadressat erfüllen würde, eine Qualifikation oder Privilegierung des vom Tatgenossen verwirklichten Tatbestandes ist. Zwar handelt es sich bei der Mittäterschaft ihrem Rechtsgrund nach um keine akzessorische Beteiligungsform im normativen Sinne. Das gefundene Ergebnis folgt jedoch daraus, dass der Wortlaut des § 25 II StGB die gemeinschaftliche Begehung einer Straftat fordert. Die Tatbestandsbezogenheit der Mittäterschaft ist Konsequenz der Wechselseitigkeit der außerordentlichen Verhaltenszurechnung.

Liegen die Voraussetzungen der teilweisen Mittäterschaft nicht vor, kann ungeachtet dessen auf die mittelbare Täterschaft gemäß § 25 I 2. Var StGB zurückgegriffen werden, da diese auch Konstellationen erfasst, in denen der Vordermann die Tat im Auftrag des Hintermanns für diesen begeht. Vergegenwärtigt man sich noch einmal die vorgestellten Fälle, würde die außerordentliche Verhaltenzurechnung in umgekehrter Richtung als die hier problematisierte im Rahmen der Mittäterschaft ohnehin leerlaufen, da die betreffenden Beteiligten in ihrer Person keinen bzw. keinen ausreichenden Tatbeitrag erbracht haben. Und ohnehin hat die Mittäterschaft - schließt man sich der hier vertretenen Konzeption an - eigentlich einen juristisch relevanten Anwendungsbereich nur dann, wenn jeder der Tatgenossen einen Teil des Tatbestandes verwirklicht. ${ }^{81}$ Auch insoweit ist der Rückgriff auf die mittelbare Täterschaft dogmatisch der ehrlichere Weg!

81 Haas, Matt/Renzikowski-StGB, (Fn. 57), § 25 Rn. 81. 